\title{
Geriatric Nutritional Risk Index as a Simple Predictor of Mortality in Maintenance Hemodialysis Patients: A Single Center Study
}

\author{
Sayaka Takahashi', Keiko Suzuki' ${ }^{1}$, Fumiko Kojima1, Yoshiko Tanaka1, Kosaku Nitta ${ }^{2 *}$ \\ ${ }^{1}$ Department of Nephrology, Shinjuku Ishikawa Clinic \\ ${ }^{2}$ Department of Medicine, Kidney Center, Tokyo Women's Medical University, Tokyo, Japan \\ Email: ${ }^{*}$ knitta@kc.twmu.ac.jp
}

Received 30 March 2015; accepted 19 May 2015; published 22 May 2015

Copyright @ 2015 by authors and Scientific Research Publishing Inc.

This work is licensed under the Creative Commons Attribution International License (CC BY). http://creativecommons.org/licenses/by/4.0/

(c) (i) Open Access

\begin{abstract}
Background: Malnutrition is associated with higher risk of mortality in maintenance hemodialysis (MHD) patients. The geriatric nutritional risk index (GNRI) has been developed as a tool to assess the nutritional risk. Objectives: The purpose of the present study was to examine the significance of the GNRI as a mortality predictor in MHD patients. Methods: We retrospectively examined the GNRI of 259 MHD patients aged $59.2 \pm 12.8$ years, and followed up for 36 months. The patients were divided into two groups according to GNRI values of 91.0. Predictors for all-cause mortality were examined using Kaplan-Meier and Cox proportional-hazards analyses. Results: During the follow-up period of 36 months, a total of 76 patients died. Kaplan-Meier survival analysis showed that the subjects with a GNRI < $91(n=29)$ had a lower survival rate than those with a GNRI > $91(n$ = 230) (Log-rank test, $p<0.0001$ ). Multivariate Cox proportional-hazards analysis demonstrated that the GNRI was a significant predictor of adjusted all-cause mortality (hazard ratio 0.927; confidence interval $0.870-0.984, p=0.013$ ). Conclusion: The results of the present study demonstrate that the GNRI is a strong predictor of all-cause mortality in MHD patients.
\end{abstract}

\section{Keywords}

Hemodialysis, Malnutrition, Geriatric Nutritional Risk Index, Mortality

\section{Introduction}

Protein-energy wasting (PEW) is an important issue in hemodialysis (HD) patients, with a reported prevalence

${ }^{*}$ Corresponding author.

How to cite this paper: Takahashi, S., Suzuki, K., Kojima, F., Tanaka, Y. and Nitta, K. (2015) Geriatric Nutritional Risk Index as a Simple Predictor of Mortality in Maintenance Hemodialysis Patients: A Single Center Study. International Journal of Clinical Medicine, 6, 354-362. http://dx.doi.org/10.4236/ijcm.2015.65046 
ranging from about $20 \%$ to $78 \%$ [1]. Nutritional risk factor is one of the strong predictors of mortality in HD patients [2] [3]. Thus, evaluation of nutritional status is essential clinical procedure for managing these patients.

Multiple mechanisms have been reported to explain the link between PEW and mortality in HD patients, including derangements in muscle, adipose tissue, gastrointestinal, hematopoietic and immune systems, abnormal activation of the inflammatory process [4]. A clinical assessment of nutritional status is mandatory in HD patients, but there is no single gold-standard method, easily performed in a reproducible manner and not affected by other confounding conditions. Therefore, it is necessary to screen patients for the presence of PEW with a variety of measures and to perform this investigation on a regular clinical practice [5].

The geriatric nutritional risk index (GNRI) is a very simple method based on body weight, height and serum albumin levels to assess nutritional status in various pathological conditions. Previous studies have investigated the reliability of GNRI in assessing malnutrition [6] and in predicting all-cause mortality in chronic HD patients [7] [8]. All the reported studies in uremic patients were performed, however, in Asian cohorts and data from European, or Caucasian, populations are lacking.

The purpose of the present study was to evaluate the GNRI as a nutritional tool for predicting mortality in a Japanese MHD population.

\section{Methods}

\subsection{Study Subjects and Procedures}

This was a retrospective cohort study conducted at a single center in Japan. The subjects were recruited from among patients who had been routinely treated through an arteriovenous fistula in the dialysis unit of the Shinjuku Ishikawa Clinic for at least 6 months. The Institutional Review Board of the Shinjuku Ishikawa Clinic approved all study protocols, and they were performed in accordance with the Declaration of Helsinki guidelines regarding ethical principles for medical research involving human subjects. Informed consent was obtained from all of the subjects.

HD patients with malignancy, active inflammation, liver cirrhosis, gastrointestinal bleeding, or severe illness were excluded from participation. The patients who were enrolled as subjects $(n=259)$ underwent stable regular HD with a bicarbonate dialysate. Their underlying diseases were chronic glomerulonephritis $(n=98)$, diabetic nephropathy $(n=76)$, hypertensive nephrosclerosis $(n=52)$, polycystic kidney disease $(n=18)$, and chronic pyeonephritis $(n=9)$, or unknown origin $(n=6)$.

All patients were on thrice-weekly HD and no further selection was performed in patients. Blood pressure (BP) was measured with a mercury sphygmomanometer with the patient in the supine position after resting for 10 to 15 minutes, and mean values for the 1-month period preceding enrollment were used in the statistical analysis. Dry weight was targeted to achieve a normotensive edema-free state. Previous cardiovascular disease and smoking status were collected from medical records. Diabetes was defined as a history or presence of diabetes and/or a fasting plasma glucose concentration $>126 \mathrm{mg} / \mathrm{dl}$ or HbA1c concentration $>6.5 \%$ or prescription of glucose-lowering agents.

\subsection{Laboratory and Nutritional Parameters}

Blood sampling was performed before the mid-week dialysis session day after an overnight fast. Serum urea nitrogen, creatinine, calcium, phosphorous, albumin, total cholesterol, high-density lipoprotein (HDL)-cholesterol and C-reactive protein (CRP) levels and the hemoglobin concentration were measured with an autoanalyzer (Hitachi Co., Tokyo, Japan) by standard laboratory methods. Total calcium was corrected by the patient's albumin level. Intact parathyroid hormone (iPTH) was measured by an immunoradiometric assay (Nichol's Institute, San Juan Capistrano, CA, USA). The body mass index (BMI) was expressed in $\mathrm{kg} / \mathrm{m}^{2}$. Urea kinetics were assessed by measuring a blood-based dialysis parameter, $\mathrm{Kt} / \mathrm{V}$ [9], and the mean value of the 3 measurements during each of the 3 months before the start of the study was used in the analysis. The normalized protein catabolism rate (nPCR) was used as an indirect indicator of protein intake and was obtained using the following formula as previously described [10].

The GNRI was calculated by modifying the Nutritional risk index for elderly patients, as reported by Yamada et al. [6] as follows: GNRI $=[14.89 \times$ albumin $(\mathrm{g} / \mathrm{dl})]+[41.7 \times($ body weight/ideal body weight) $]$. As body weight we considered the value at the end of the dialysis session, and it was used also for BMI calculation. Body 
weight/ideal body weight was set to 1 when the patient's body weight exceeded the ideal body weight [6]. The ideal body weight in the present study was calculated using height and a BMI of 22, which is reportedly associated with the lowest morbidity rate in the Asian population [11].

\subsection{Study Outcome}

Data for endpoints were obtained from hospital charts. Primary endpoint of the study was all-cause mortality during the follow-up period from January 1, 2011, to December 31, 2014. The vital status of the subjects was assessed by searching the electronic dialysis records. Patients were censored if they were alive on December 31 , 2014.

\subsection{Statistical Analysis}

Normally distributed, unpaired continuous values were expressed as means \pm SD and compared by performing an analysis of variance. Nonparametric values were expressed as median values and compared by performing the Kruskal-Wallis test. Categorical values were expressed as percentages and compared by performing the Fisher's exact test. Logistic regression analysis was used in a model to determine how independent variables predicted the GNRI value. We considered some variables that possess P-value $<0.10$ in univariate logistic regression analyses, gender, dialysis vintage, and presence of diabetes as independent variables for multivariate logistic regression analyses. The area under the receiver operating curve (ROC) analysis was performed to calculate the cut-off values of GNRI.

The survival analysis was based on the Kaplan-Meier curve with subjects censored for death. A log-rank test was used to compare the survival rates of two groups. A multivariate Cox proportional hazards model with adjustment for multivariate factors was used to evaluate mortality risk. Results were expressed as a hazard ratio (HR) with 95\% confidence intervals (CIs). A P-value < 0.05 was considered to be statistically significant. All statistical analyses were performed by using the SAS version 9.2 software program (SAS Institute Inc., Cary, NC, USA) for Windows personal computers.

\section{Results}

The baseline characteristics of the study population are shown in Table 1 . The mean age of the 259 subjects was $59.2 \pm 12.8$ years, and the mean dialysis vintage was 131.3.2 \pm 92.0 years months. The prevalence of diabetes $68.0 \%$ in all subjects. The study population included 70 women and 189 men. To determine a possible cut-off value of GNRI for survival, the sensitivity and specificity were examined by ROC analysis. A GNRI value of 91 was shown to indicate the highest value for sensitivity and specificity (Figure 1). The AUC for GNRI from ROC analysis was 0.673 (95\% CI, 0.545 - 0.781) and the positive likelihood for GNRI was 4.719.

The clinical characteristics of the 259 subjects according to GNRI are shown in Table 2. The mean GNRI value of the subjects with a GNRI $<91$ was $87.6 \pm 3.3$, and that of the subjects with a GNRI $>91$ was $101.8 \pm$ 7.4. Subjects with a GNRI < 91 exhibited significantly older age, lower body weight and BMI, lower serum albumin and creatinine compared with those with a GNRI $>91$. As shown in Figure 2, there was a significant correlation between age and GNRI ( $\mathrm{r}=-0.3515, \mathrm{P}<0.0001)$.

During the follow-up period of 36 months, a total of 26 patients died. The Kaplan-Meier analysis revealed that subjects with a GNRI $<91(n=29)$ had a significant lower survival rate compared with that of those with a GNRI > $91(\mathrm{n}=230)(\mathrm{P}<0.0001$, Figure 3). Univariate Cox proportional hazards analysis for mortality showed that age (HR 1.079, 95\% CI 1.042 - 1.119, P < 0.0001), serum levels of albumin (HR 0.185, 95\% CI 0.065 0.580, P = 0.0043), creatinine (HR 0.720, 95\% CI $0.612-0.846, \mathrm{P}<0.0001$ ) and calcium (HR 1.979, 95\% CI 1.091 - 3.577, $\mathrm{P}=0.0245$ ) were significant predictor of mortality (Table 3). Multivariate Cox proportional hazards analysis demonstrated that GNRI was a significant predictor of mortality (HR 0.927, 95\% CI 0.870 - 0.984 , $\mathrm{P}=0.0130$ ) (Table 4). In addition, age (HR 1.063, 95\% CI $1.023-1.107, \mathrm{P}=0.0016)$, Kt/V (HR 0.058, 95\% CI $0.005-0.682, \mathrm{P}=0.0233$ ) and serum calcium levels (HR 1.819, 95\% CI $1.090-3.004, \mathrm{P}=0.0226$ ) were also significant predictors of mortality.

\section{Discussion}

We investigated the GNRI as a nutritional tool for predicting mortality in a Japanese MHD population. We used 


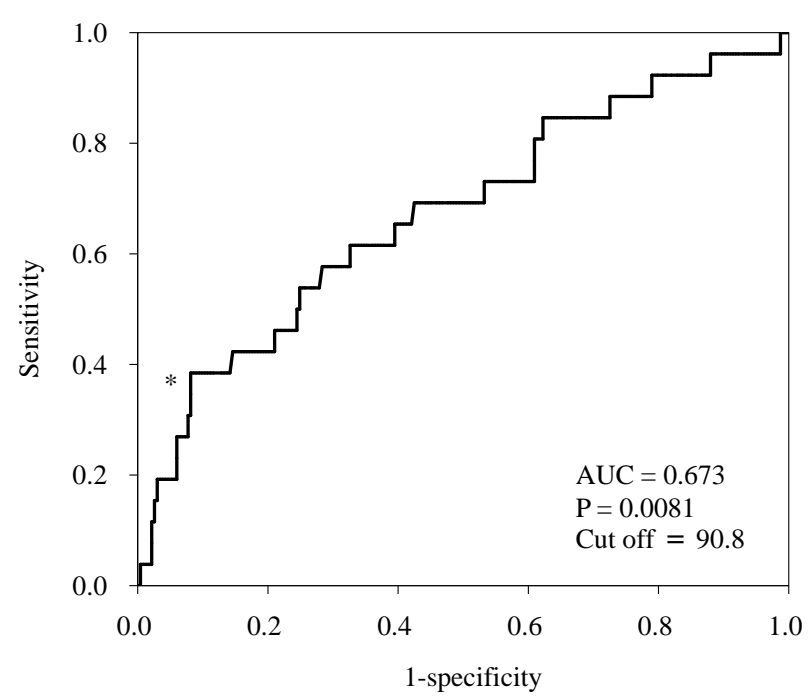

Figure 1. Receiver operating curve analysis to examine a cutoff value of GNRI for all-cause mortality.

Table 1. Baseline characteristics of the study population.

\begin{tabular}{|c|c|}
\hline Variable & $\mathrm{N}=259$ \\
\hline Age (years) & $59.2 \pm 12.8$ \\
\hline Men/women & $189 / 70$ \\
\hline Dialysis vintage (months) & $131.3 \pm 92.0$ \\
\hline Diabetes $(-/+)$ & $176 / 83$ \\
\hline Body weight (kg) & $60.2 \pm 13.5$ \\
\hline Body mass index & $22.3 \pm 3.7$ \\
\hline Albumin (g/dl) & $3.9 \pm 0.3$ \\
\hline Blood urea nitrogen (mg/dl) & $68.0 \pm 14.9$ \\
\hline Creatinine (mg/dl) & $11.9 \pm 2.4$ \\
\hline C-reactive protein (mg/dl) & $0.30 \pm 0.44$ \\
\hline GNRI & $100.2 \pm 8.4$ \\
\hline $\mathrm{Kt} / \mathrm{V}$ & $1.45 \pm 0.24$ \\
\hline Systolic blood pressure (mmHg) & $152.1 \pm 22.6$ \\
\hline Diastolic blood pressure (mmHg) & $82.4 \pm 14.6$ \\
\hline Normalized PCR & $0.93 \pm 0.17$ \\
\hline Hemoglobin (g/dl) & $10.6 \pm 0.9$ \\
\hline Ferritin (ng/ml) & $72.7 \pm 75.6$ \\
\hline Calcium (mg/dl) & $9.0 \pm 0.7$ \\
\hline Phosphorus (mg/dl) & $5.4 \pm 1.2$ \\
\hline Intact PTH (pg/ml) & $76.2 \pm 66.8$ \\
\hline Uric acid & $7.5 \pm 1.1$ \\
\hline Total cholesterol (mg/dl) & $158.9 \pm 31.8$ \\
\hline HDL-cholesterol (mg/dl) & $47.3 \pm 14.9$ \\
\hline Non-HDL-cholesterol (mg/dl) & $111.6 \pm 31.7$ \\
\hline
\end{tabular}

GNRI, geriatric nutritional risk index; PCR, protein catabolic rate; PTH, parathyroid hormone. 


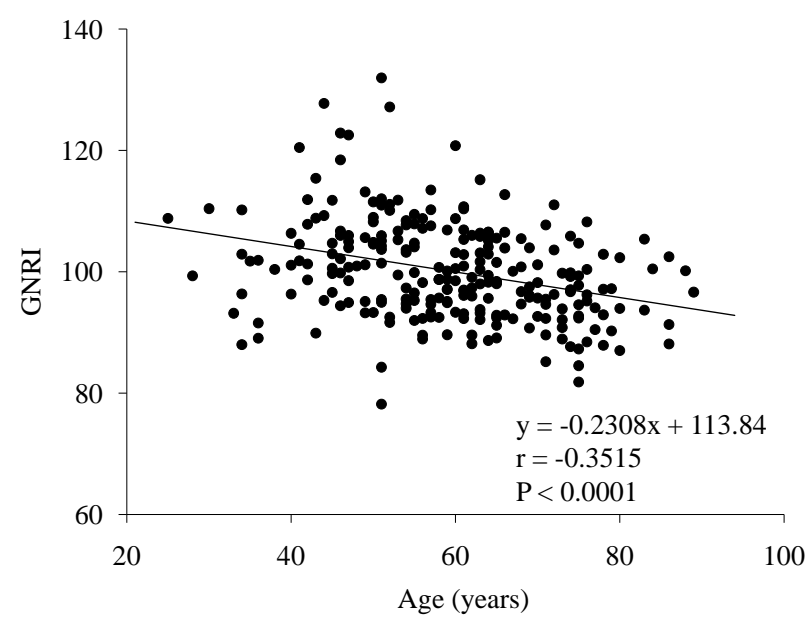

Figure 2. Relationship between age and GNRI.

Table 2. Clinical characteristics of 259 study subjects according to geriatric nutritional risk index (GNRI).

\begin{tabular}{|c|c|c|c|}
\hline & GNRI $<91$ & GNRI $\geq 91$ & $\mathrm{P}$ \\
\hline Age (years) & $65.8 \pm 13.4$ & $58.4 \pm 12.5$ & 0.0029 \\
\hline Men/women & $16 / 13$ & $173 / 57$ & 0.0274 \\
\hline Dialysis vintage (months) & $147.0 \pm 95.2$ & $129.3 \pm 91.6$ & 0.3303 \\
\hline Diabetes $(-/+)$ & $21 / 8$ & $155 / 75$ & 0.6762 \\
\hline Body weight (kg) & $46.9 \pm 8.5$ & $61.9 \pm 13.0$ & $<0.0001$ \\
\hline Body mass index & $18.2 \pm 1.8$ & $22.9 \pm 3.6$ & $<0.0001$ \\
\hline Albumin (g/dl) & $3.6 \pm 0.3$ & $3.9 \pm 0.3$ & $<.0001$ \\
\hline Blood urea nitrogen (mg/dl) & $64.6 \pm 15.3$ & $68.5 \pm 14.8$ & 0.1843 \\
\hline Creatinine (mg/dl) & $9.8 \pm 2.3$ & $12.1 \pm 2.3$ & $<0.0001$ \\
\hline C-reactive protein (mg/dl) & $0.32 \pm 0.44$ & $0.30 \pm 0.45$ & 0.8314 \\
\hline GNRI & $87.6 \pm 3.3$ & $101.8 \pm 7.4$ & $<0.0001$ \\
\hline $\mathrm{Kt} / \mathrm{V}$ & $1.6 \pm 0.3$ & $1.4 \pm 0.2$ & 0.0194 \\
\hline Systolic blood pressure (mmHg) & $147.0 \pm 28.3$ & $152.7 \pm 21.7$ & 0.2018 \\
\hline Diastolic blood pressure (mmHg) & $78.7 \pm 14.7$ & $82.9 \pm 14.6$ & 0.1497 \\
\hline Normalized PCR & $0.92 \pm 0.18$ & $0.93 \pm 0.17$ & 0.5988 \\
\hline Hemoglobin (g/dl) & $10.4 \pm 1.2$ & $10.6 \pm 0.8$ & 0.1935 \\
\hline Ferritin (ng/ml) & $95.1 \pm 83.2$ & $69.9 \pm 74.3$ & 0.0966 \\
\hline Calcium (mg/dl) & $8.9 \pm 0.9$ & $9.0 \pm 0.6$ & 0.5181 \\
\hline Phosphorus (mg/dl) & $5.1 \pm 1.5$ & $5.5 \pm 1.2$ & 0.1140 \\
\hline Intact PTH (pg/ml) & $86.9 \pm 75.2$ & $74.8 \pm 65.7$ & 0.3590 \\
\hline Uric acid (mg/dl) & $7.2 \pm 1.0$ & $7.5 \pm 1.1$ & 0.1583 \\
\hline Total cholesterol (mg/dl) & $157.2 \pm 34.7$ & $159.2 \pm 31.5$ & 0.7522 \\
\hline HDL-cholesterol (mg/dl) & $49.6 \pm 17.1$ & $47.1 \pm 14.7$ & 0.3849 \\
\hline Non-HDL-cholesterol (mg/dl) & $107.6 \pm 34.5$ & $112.1 \pm 31.4$ & 0.4680 \\
\hline
\end{tabular}




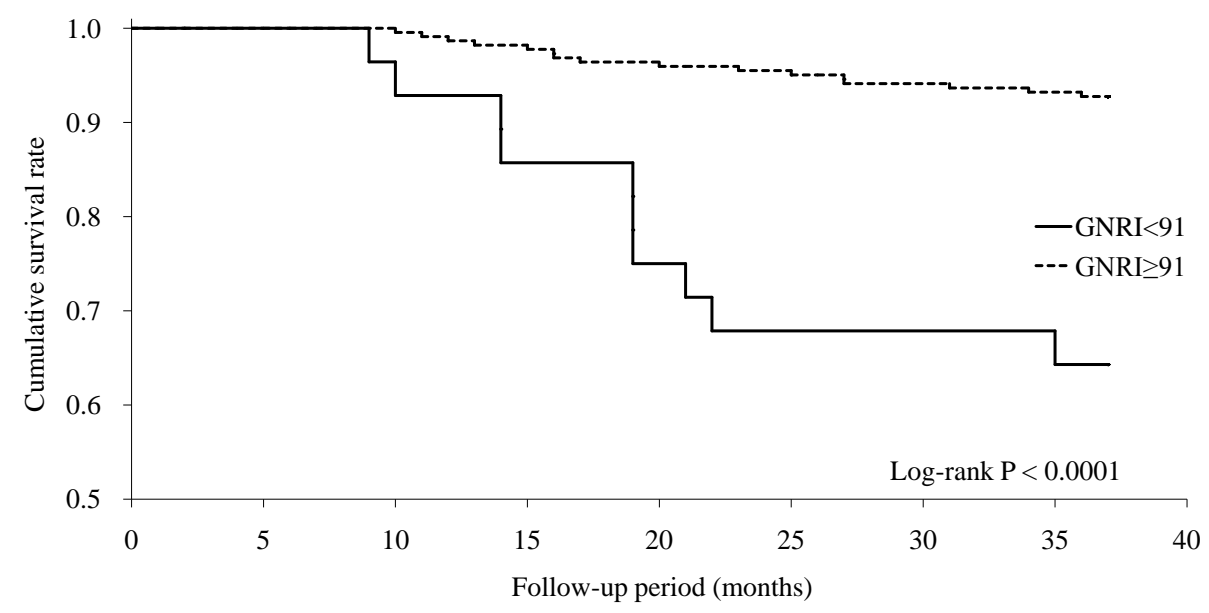

Figure 3. Kaplan-Meier survival curves for all-cause mortality according to geriatric nutritional risk index values.

Table 3. Univariate Cox proportional hazards analysis of mortality.

\begin{tabular}{|c|c|c|c|}
\hline & HR & $95 \% \mathrm{CI}$ & $\mathrm{P}$ \\
\hline Age (/years) & 1.079 & $1.042-1.119$ & $<0.0001$ \\
\hline Men (vs. women) & 1.534 & $0.625-4.595$ & 0.3697 \\
\hline Dialysis vintage (/months) & 1.003 & $0.999-1.006$ & 0.1855 \\
\hline Diabetes (vs. No) & 1.187 & $0.506-2.603$ & 0.6806 \\
\hline Body weight (/kg) & 0.978 & $0.945-1.008$ & 0.1585 \\
\hline Body mass index (/1) & 0.922 & $0.814-1.031$ & 0.1629 \\
\hline Albumin (g/dl) & 0.185 & $0.065-0.580$ & 0.0043 \\
\hline Blood urea nitrogen (/mg/dl) & 0.984 & $0.958-1.010$ & 0.2339 \\
\hline Creatinine (/mg/dl) & 0.720 & $0.612-0.846$ & $<0.0001$ \\
\hline C-reactive protein (/mg/dl) & 1.416 & $0.655-2.378$ & 0.3246 \\
\hline GNRI (/1) & 0.928 & $0.880-0.977$ & 0.0037 \\
\hline $\mathrm{Kt} / \mathrm{V}(/ 1)$ & 0.225 & $0.040-1.227$ & 0.0854 \\
\hline Systolic blood pressure (/mmHg) & 1.011 & $0.993-1.028$ & 0.2326 \\
\hline Diastolic blood pressure (/mmHg) & 0.991 & $0.965-1.017$ & 0.4789 \\
\hline Normalized PCR (/1) & 0.145 & $0.013-1.473$ & 0.1035 \\
\hline Hemoglobin (/g/dl) & 1.126 & $0.728-1.814$ & 0.6100 \\
\hline Ferritin (/ng/ml) & 1.003 & $0.999-1.006$ & 0.1488 \\
\hline Calcium (/mg/dl) & 1.979 & $1.091-3.577$ & 0.0245 \\
\hline Phosphorus (/mg/dl) & 0.765 & $0.539-1.063$ & 0.1135 \\
\hline Intact PTH $(/ \mathrm{pg} / \mathrm{ml})$ & 1.004 & $0.999-1.008$ & 0.1307 \\
\hline Uric acid (/mg/dl) & 0.885 & $0.610-1.253$ & 0.5009 \\
\hline Total cholesterol (/mg/dl) & 0.997 & $0.985-1.009$ & 0.6601 \\
\hline HDL-cholesterol (/mg/dl) & 0.973 & $0.942-1.001$ & 0.0571 \\
\hline Non-HDL-cholesterol (/mg/dl) & 1.002 & $0.990-1.014$ & 0.6795 \\
\hline
\end{tabular}


Table 4. Multivariate Cox proportional hazards analysis of mortality.

\begin{tabular}{cccc}
\hline & HR & 95\%CI & P \\
\hline Age (/years) & 1.063 & $1.023-1.107$ & 0.0016 \\
Men (vs. women) & 1.228 & $0.425-4.186$ & 0.7175 \\
Dialysis vintage (/months) & 1.003 & $0.998-1.007$ & 0.2143 \\
Diabetes (vs. No) & 1.181 & $0.479-2.774$ & 0.7081 \\
GNRI (/1) & 0.927 & $0.870-0.984$ & 0.0130 \\
Kt/V (/1) & 0.058 & $0.005-0.682$ & 0.0233 \\
Calcium (/mg/dl) & 1.819 & $1.090-3.004$ & 0.0226 \\
\hline
\end{tabular}

a cut-off value of GNRI of 91 to identify subjects with the highest risk according to ROC analysis. Subjects with a GNRI $<91$ had a significant lower survival rate compared with that of those with a GNRI $>91$. The results of the present study clearly showed that the GNRI value represents a strong predictor for all-cause mortality in Japanese HD patients.

Assessment of nutritional status is essential to prevent, diagnose and treat uremic malnutrition in uremic patients [5]. Biochemistry, anthropometry, body composition analysis, and interviews are variably used together with scores and indexes to obtain a comprehensive assessment of the nutritional status in uremic patients [5]. Many nutritional screening tools have been reported in the elderly, hospitalized patients, community patients, or patients with cancer or infections. Some of them may be safely and easily applied to MHD patients as well [12]. The Subjective Global Nutritional Assessment is a well validated clinical tool for screening malnutrition [2] and the malnutrition-inflammation score (MIS) is able to predict mortality and hospitalization in MHD patients [13]. However, both require subjective assessment and judgment by a skilled investigator.

In the present study, GNRI was one of the parameters as a predictor of all-cause mortality. The GNRI consists of few objective components, including serum albumin and BMI and represents a simple nutritional screening tool [6]. The GNRI score has been shown able to predict increased healthcare costs and higher risk of hospitalization in independent-living older adults; so it may be a low-cost tool that might be routinely used in population-based settings [14].

Yamada et al. [6] suggested that the most accurate GNRI cut-off value to identify malnourished HD patients was $<91.2$, based on the MIS. Kobayashi et al. [7] reported that HD patients with GNRI $<90$ exhibited a poorer nutritional status in terms of a lower BMI, serum levels of albumin and creatinine compared with GNRI > 90 . Park et al. [8] confirmed in Korean HD patients that GNRI may be a significant predictor of mortality. More recently, Panichi et al. [15] have shown that values lower than 92 may represent a strong indicator of unfavorable outcome in Caucasian HD patients.

Takahashi et al. have recently indicated that GNRI at initiation of HD therapy could predict cardiovascular mortality with incremental value of the predictability compared to serum albumin and body mass index in HD patients [16]. Although the lowest GNRI quartile $(<92)$ is strongly associated with malnutrition signs and with increased risk of all-cause mortality, no predictive value emerged regarding non-fatal cardiovascular events in HD patients. This apparent discrepancy may be explained considering that malnourished patients have lower survival and so less chance to develop a cardiovascular event, but when a cardiovascular event does occur, a malnourished patient has less chance to recover and more chance to die. Our data also clearly confirmed the reliability of GNRI as a tool for the diagnosis of malnutrition in a longitudinal observation [17].

Various mechanisms may explain the link between PEM and mortality in renal failure, including derangements in muscle, adipose tissue, gastrointestinal, hematopoietic and immune systems, and abnormal activation of the inflammatory process [18], as well as co-morbidities. Low albumin, cholesterol, and BMI are indicators of PEM, but they may not be causally responsible for the negative outcome. The reduction in muscle mass, namely sarcopenia, observed in PEM may be due to uremic toxins or procatabolic conditions (metabolic, hormonal, or neuropathic derangements, including inactivity).

There are some limitations in this study that should be considered. First, this study is based on a relatively small sample size of HD patients from a single center, limiting the ability of our findings to be generalized. 
Second, this study used only a retrospective approach, without manipulation of confounding factors. We could not rule out a recalling bias. Randomized, controlled trials are needed to explore the role of GNRI value on mortality risk in chronic HD patients. Third, we did not show the prescription of renin-angiotensin system inhibitors, statins, and vitamin D. These agents may affect the mortality risk of GNRI. Fourth, other confounding factors may be neglected because the GNRI was calculated from the formula including serum albumin and BMI.

\section{Conclusion}

The results of the present study clearly show that GNRI values are significantly associated with all-cause mortality in Japanese HD patients. We conclude that the GNRI may be a very simple tool to use that may be considered as a reliable predictor for all-cause mortality risk in the HD population.

\section{Acknowledgements}

The authors are very grateful to medical staffs of Shinjuku Ishikawa Clinic who provided high-quality data. This study was in part supported by a grant from Japan Promotion Society for Cardiovascular Disease.

\section{Disclosure}

The authors have no conflicts of interest to declare.

\section{References}

[1] Bouillanne, O., Morineau, G., Dupont, C., Coulombel, I., Vincent, J.P., Nicolis, S., et al. (2005) Geriatric Nutritional Risk Index: A New Index for Evaluating At-Risk Elderly Medical Patients. American Journal of Clinical Nutrition, 82, 777-783.

[2] de Mutsert, R., Grootendorst, D.C., Axelsson, J., Boeschoten, E.W., Krediet, R.T. and Dekker, F.W., NECOSAD Study Group (2008) Excess Mortality to Interaction between Protein-Energy Wasting, Inflammation and Cardiovascular Disease in Chronic Dialysis Patients. Nephrology Dialysis Transplantation, 23, 2957-2964. http://dx.doi.org/10.1093/ndt/gfn167

[3] Fouque, D., Kalantar-Zadeh, K., Kopple, J., Cano, N., Cuppari, L., Franch, H., et al. (2008) A Proposed Nomenclature and Diagnostic Criteria for Protein-Energy Wasting in Acute and Chronic Kidney Disease. Kidney International, 73, 391-398. http://dx.doi.org/10.1038/sj.ki.5002585

[4] Kovesdy, C.P. and Kalantar-Zadeh, K. (2009) Why Is Protein-Energy Wasting Associated with Mortality in Chronic Kidney Disease? Seminars in Nephrology, 29, 3-14. http://dx.doi.org/10.1016/j.semnephrol.2008.10.002

[5] Pupin, L.B. and Ikizler, T.A. (2004) Assessment and Monitoring of Uremic Malnutrition. Journal of Renal Nutrition, 14, 6-19. http://dx.doi.org/10.1053/j.jrn.2003.10.001

[6] Yamada, K., Furuya, R., Takita, T., Maruyama, Y., Yamaguchi, Y., Ohkawa, S., et al. (2008) Simplified Nutritional Screening Tools for Patients on Maintenance Hemodialysis. American Journal of Clinical Nutrition, 87, 106-113.

[7] Kobayashi, I., Ishimura, E., Kato, Y., Okuno, S., Yamamoto, T., Yamakawa, T., et al. (2010) Geriatric Nutritional Risk Index, a Simplified Nutritional Screening Index, Is a Significant Predictor of Mortality in Chronic Dialysis Patients. Nephrology Dialysis Transplantation, 25, 3361-3365. http://dx.doi.org/10.1093/ndt/gfq211

[8] Park, J.H., Kim, S.B., Shin, H.S., Jung, Y.S. and Rim, H. (2012) Geriatric Nutritional Risk Index May Be a Significant Predictor of Mortality in Korean Hemodialysis Patients: A Single Center Study. Therapeutic Apheresis and Dialysis, 16, 121-126. http://dx.doi.org/10.1111/j.1744-9987.2011.01046.x

[9] Daugirdas, J.T. (1993) Second Generation Logarithmic Estimates of Single-Pool Variable Volume Kt/V: An Analysis of Error. Journal of the American Society of Nephrology, 4, 1205-1213.

[10] Shinzato, T., Nakai, S., Fujita, Y., Takai, I., Morita, H., Nakane, K., et al. (1994) Determination of Kt/V and Protein Catabolic Rate Using Pre- and Postdialysis Blood Urea Nitrogen Concentrations. Nephron, 67, 280-290. http://dx.doi.org/10.1159/000187980

[11] Examination Committee of Criteria for “Obesity Disease” in Japan. Japan Society for the Study of Obesity (2002) New Criteria for “Obesity Disease” in Japan. Circulation Journal, 66, 987-992. http://dx.doi.org/10.1253/circj.66.987

[12] Kalantar-Zadeh, K., Kopple, J.D., Humphreys, M.H. and Block, G. (2004) Comparing Outcome Predictability of Markers of Malnutrition-Inflammation Complex Syndrome in Hemodialysis Patients. Nephrology Dialysis Transplantation, 19, 1507-1519. http://dx.doi.org/10.1093/ndt/gfh143

[13] Kalantar-Zadeh, K., Kopple, J.D., Block, G. and Humphreys, M.H. (2001) A Malnutrition-Inflammation Score Is Cor- 
related with Morbidity and Mortality in Maintenance Hemodialysis Patients. American Journal of Kidney Disease, 38, 1251-1263. http://dx.doi.org/10.1053/ajkd.2001.29222

[14] Baumeister, S.E., Fischer, B., Doring, A., Koenig, W., Zierer, A., John, J., et al. (2011) The Geriatric Nutritional Risk Index Predicts Increased Healthcare Costs and Hospitalization in a Cohort of Community-Dwelling Older Adults: Results from the MONICA/KORA Augsburg Cohort Study, 1994-2005. Nutrition, 27, 534-542. http://dx.doi.org/10.1016/j.nut.2010.06.005

[15] Panichi, V., Cupisti, A., Rosati, A., Di Giorgio, A., Scatena, A., Menconi, O., et al. (2014) Geriatric Nutritional Risk Index Is a Strong Predictor of Mortality in Hemodialysis Patients: Data from the Riscavid Cohort. Journal of Nephrology, 27, 193-201. http://dx.doi.org/10.1007/s40620-013-0033-0

[16] Takahashi, H., Ito, Y., Ishii, H., Aoyama, T., Kamoi, D., Kasuga, H., et al. (2014) Geriatric Nutritional Risk Index Accurately Predicts Cardiovascular Mortality in Incident Hemodialysis Patients. Journal of Cardiology, 64, 32-36. http://dx.doi.org/10.1016/j.jjcc.2013.10.018

[17] Beberashvili, I., Azar, A., Sinuani, I., Kadoshi, H., Shapiro, G., Feldman, L., et al. (2012) Comparison Analysis of Nutritional Scores for Serial Monitoring of Nutritional Status in Hemodialysis Patients. Clinical Journal of American Society of Nephrology, 8, 443-451. http://dx.doi.org/10.2215/CJN.04980512

[18] Carrero, J.J., Stenvinkel, P., Cuppari, L., Ikizler, T.A., Kalantar-Zadeh, K., Kaysen, G., et al. (2013) Etiology of the Protein-Energy Wasting Syndrome in Chronic Kidney Disease: A Consensus Statement from the International Society of Renal Nutrition and Metabolism (ISRNM). Journal of Renal Nutrition, 23, 77-90. http://dx.doi.org/10.1053/j.jrn.2013.01.001 